\title{
Solid-State Cavity Quantum Electrodynamics Using Quantum Dots
}

\author{
J.M. Gérard, B. Gayral, E. Moreau, I. Robert
}

AND I. ABRAM

CNRS/Laboratoire de Photonique et de Nanostructures 196 av. H. Ravera, 92225 Bagneux Cedex, France

\begin{abstract}
We review the recent development of solid-state cavity quantum electrodynamics using single self-assembled In As quantum dots and three-dimensional semiconductor microcavities. We discuss first prospects for observing a strong coupling regime for single quantum dots. We then demonstrate that the strong Purcell effect observed for single quantum dots in the weak coupling regime allows us to prepare emitted photons in a given state (the same spatial mode, the same polarization). We present finally the first single-mode solid-state source of single photons, based on an isolated quantum dot in a pillar microcavity. This optoelectronic device, the first ever to rely on a cavity quantum electrodynamics effect, exploits both Coulomb interaction between trapped carriers in a single quantum dot and single mode photon funneling by the microcavity.
\end{abstract}

PACS numbers: $42.50 . D_{v}, 78.55 . \mathrm{Cr}$

\section{Introduction}

Cavity quantum electrodynamics (CQED) has become since 1990 a major source of inspiration for basic research in optoelectronics [1]. In the 80's, a beautiful series of experiments on atoms in microwave and optical cavities had demonstrated that optical processes including spontaneous emission (SE) can be deeply modified by using a cavity to tailor the emitter-field coupling [2]. Among other effects observable in the low- $Q$ CQED regime, the modification of the emission diagram, the enhancement or inhibition of the SE rate, the funneling of SE photons into a single mode and the control of the SE process on the single photon level are particularly attractive to improve optoelectronic devices or develop the novel ones. For very high- $Q$ (i.e. weakly damped) cavities $\mathrm{SE}$ can even become a reversible process (strong coupling regime). 
The high quality of some solid-state microcavities available until the early 90 's has permitted some major achievements, such as the strong coupling for quantum wells in planar cavities [3], the fabrication of high-efficiency microca vity LEDs which exploit SE angular redistribution [4], and low-threshold vertical-cavity surface emitting lasers [5] or microsphere lasers [6]. However, the broadness of the spectral line of usual solid-state emitters (bulk semiconductors or quantum wells, rare earth atoms,...) has been for long a major hindrance to the observation of several important CQED effects, including SE rate enhancement (so-called "Purcell effect").

Self-assembled semiconductor quantum dots (QDs) are particularly well suited for performing solid-state CQED [7], as shown by the observation of a strong Purcell effect for QDs in semiconductor microcavities [7-11]. In this review paper, we first recall briefly some relevant properties of QDs in this context, and present a vailable 3D solid-state microcavities. After discussing whether the strong coupling regime can be achieved for single QDs, we present recent experimental results obtained in the weak coupling regime. We show finally that an efficient single-mode solid-state source of single photons can be realized using isolated QDs in a pillar microcavity. This device which exploits both a few particle Coulomb effects in QDs and the Purcell effect is the first of a novel class of optoelectronic devices based on solid-state CQED.

\section{Some assets of self-assembled semiconductor QDs for quantum optics and cavity quantum electrodynamics}

Solid-state CQED experiments have been until now mostly performed on InAs/GaAs QDs in GaAs/GaAlAs microcavities. Though other QDs, such as II-VI self-assembled QDs [12,13], QDs formed by interface fluctuations in quantum wells [14] or semiconductor nanocrystals $[15,16]$ are also potentially interesting in this context, we concentrate our attention on that more mature system.

It has been known since 1985 [17] that strained-layer epitaxy can be used to build defect-free nanometer scale InAs rich clusters in GaAs which constitute QDs for electrons and holes. These self-assembled QDs support well-separated discrete electronic states and exhibit a single narrow emission line $(\ll k T)$ under weak excitation conditions. A linewidth lower than $25 \mu \mathrm{eV}$ (resolution limited) at $10 \mathrm{~K}$ has been reported [18] which permits us to exploit fully the potential of high $Q$ cavities (up to $Q \sim 20000$ typically) for $\mathrm{SE}$ control. Some homogeneous line broadening mostly due to the electron-phonon interaction is observed when the temperature is raised. Preliminary measurements by near-field optical microscopy suggest the emission linewidth of single QDs could be as large as 5 to $10 \mathrm{meV}$ at $300 \mathrm{~K}[19]$.

Due to the strong 3D electronic confinement in QDs, a few particle Coulomb effects dominate single QD emission spectra under high excitation conditions 


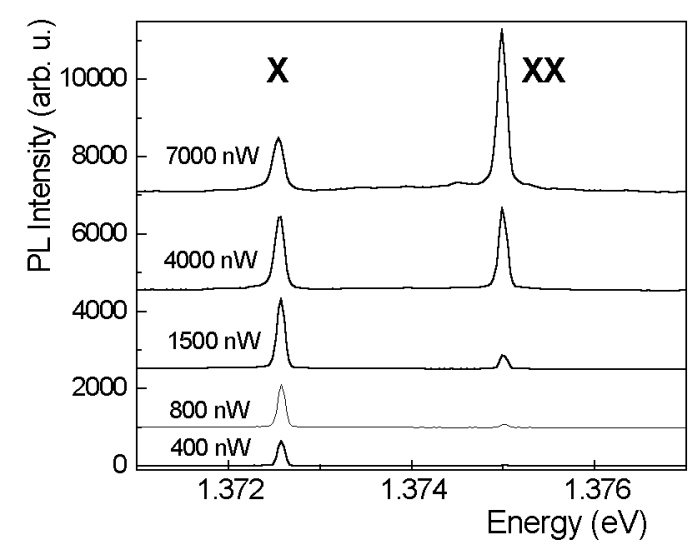

Fig. 1. Emission spectra of a single In As QD under c.w. excitation. $X$ and $X X$ lines, which exhibit different power dependences, can be identified as the emission lines of the QD when it contains one and two electron-hole pairs, respectively.

$[20,21]$ as displayed in Fig. 1. For instance, "exciton" and "biexciton" lines, into which photons are emitted when the QD contains, respectively, one or two electron-hole pairs, are separated by typically one to three $\mathrm{meV}$ for InAs QDs. This energy separation varies from QD to QD... and from one laboratory to another, which highlights a great sensitivity of these effects to the precise shape and size of the QD. As shown in Sec. 5, these effects are essential for the generation of single photons or strongly correlated photon pairs using single QDs.

Self-assembled QDs also display a large electric dipolar moment for their fundamental optical transition, providing an efficient coupling to the electromagnetic field. Due to the compressive strain experienced by InAs, this dipole is (randomly) oriented in the $x-y$ epilayer plane. Absorption experiments on InAs QD arrays have shown that the oscillator strength per QD is of the order of ten [22]; this corresponds to values of the dipole components along the $x$ or $y$ directions $d_{x}$ (or $d_{y}$ ) around $9 \times 10^{-19} \mathrm{C} \cdot \mathrm{m}$, which is ten times larger than typical values for atoms emitting at the same wavelength range. This feature is reflected by the rather short radiative lifetime ( $\sim 1 \mathrm{~ns})$ of InAs QDs in bulk GaAs.

The peak emission wavelength of InAs QD arrays can be chosen - typically in the $0.9-1.2 \mu \mathrm{m}$ range at $10 \mathrm{~K}$ - since the average size of the QDs depends reproducibly on the growth conditions in molecular beam epitaxy [23]. Such QDs display a high radiative quantum yield $(\eta \sim 1)$ provided carrier thermoemission is negligible ( $T<100$ to $200 \mathrm{~K}$ depending on the average size). This excellent yield is due to the trapping of excitons in the defect-free QDs, which prevents a possible diffusion towards non-radiative recombination centers [24].

Though moderate, size fluctuations lead to a significant dispersion of QD bandgaps within arrays, revealed by the inhomogeneous broadening of QD arrays 
emission lines (20 to $100 \mathrm{meV}$ ). This feature constitutes at first sight a major difficulty when one wants to place a single QD in resonance with a discrete cavity mode. This difficulty can be turned to some extent into an asset. Instead of placing a single QD inside the cavity (which is in practice rather tricky), it is possible to insert a collection of a few hundred QDs, which are statistically all different thanks to size fluctuations. The detuning between the cavity mode and the center of the QD distribution can be easily adjusted so that a single QD on average is resonant with the cavity mode. Fine tuning of the resonance can then be obtained using small temperature changes [11].

Finally, let us mention a major practical advantage of QDs for applications: unlike atoms, QDs can be pumped in a non-resonant way, by exciting the GaAs barrier. This opens a route toward the development of compact electrically pumped optoelectronic devices exploiting CQED effects on QDs.

\section{Presentation of available 3D semiconductor microcavities}

Unlike the microwave spectral range, where superconducting mirrors are a vailable, lossless metallic mirrors do not exist at optical frequencies. Major efforts have been devoted in the 90's to the development of dielectric microcavities able to confine light on the wavelength scale in one or several dimensions. Two basic effects can be used, alone or in combination, for that purpose. The first one is total internal reflection, which has been exploited for long in optical fibers (1D confinement), and is used to provide 3D confinement in microspheres [6] and microdisks [8, 25] (Fig. 2b). The second one is distributed bragg reflection which is implemented

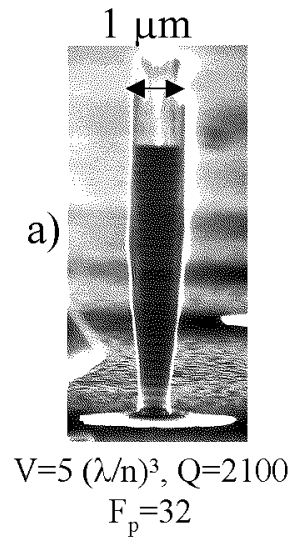

b)

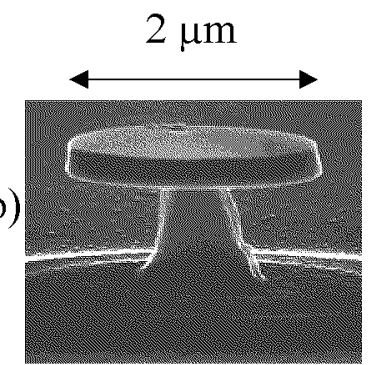

$\mathrm{V}=6(\lambda / \mathrm{n})^{3}, \mathrm{Q}=12000$

$\mathrm{F}_{\mathrm{p}}=150$

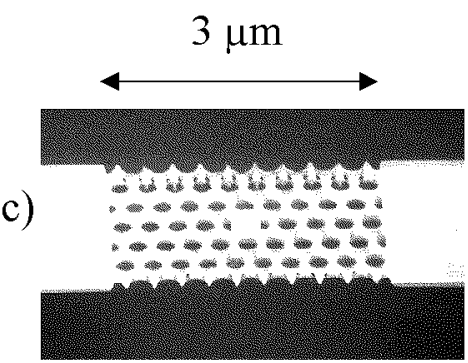

$\mathrm{V}=0.3(\lambda / \mathrm{n})^{3}, \mathrm{Q}=600$

$\mathrm{F}_{\mathrm{p}}=120$

Fig. 2. Scanning electron micrographs obtained for several 3D microca vities fabricated in our laboratory. State-of-the-art figures of merit are taken from [7] for GaAs/AlAs micropillars (a), from [8] for GaAs microdisks (b), and from [29] for 2D photonic bandgap airbridge microcavities (c). 
in 1D dielectric mirrors and more generally in photonic bandgap crystals [26]. Micropillars [27, 28] (Fig. 2a) exploit both waveguiding along the pillar axis and reflection by $1 \mathrm{D}$ Bragg mirrors to generate $3 \mathrm{D}$ confinement, whereas $2 \mathrm{D}$ photonic crystal membranes (Fig. 2c) combine a waveguiding in the semiconductor slab and lateral confinement by the photonic crystal [29].

It should be noted that these 3D dielectric microcavities do not constitute perfect "photonic boxes". Though they sustain a discrete series of 3D confined modes, they also support a continuum of leaky modes. Recent progress in the fabrication of 3D photonic crystals at optical frequencies [30] might soon change this state of affairs; whereas the density of leaky modes in the photonic bandgap energy range can be made arbitrarily low for thick crystals, a tailored defect can be used to create one (or a few) resonant cavity modes in the same energy range.

How good are 3D dielectric cavities for SE control? It is interesting to consider at this stage two basic figures of merit. The first one is the quality factor $Q$ of resonant modes, equal to the ratio of the wavelength by the mode linewidth, which describes the cavity ability for storing photons; $Q$ is simply proportional to the photon lifetime in the cavity. The second one is the effective cavity volume $V$, which reflects how strong the confinement of light is. $V$ is defined as the volume of a hypothetic cavity, defined by Born-Von Karman boundary conditions, which would provide the same maximum amplitude of the vacuum field as the cavity under study. Typical values of $Q$ and $V$ for state-of-the-art 3D semiconductor microcavities are indicated in Fig. 2. These structures exhibit a remarkable ability for concentrating light in 3D on the wavelength scale; the amplitude of the vacuum field at the antinode is for instance around $10^{5} \mathrm{~V} / \mathrm{cm}$ when $V \sim(\lambda / n)^{3}$ and $\lambda=1 \mu \mathrm{m}$ ! By contrast, $Q$ values remain moderate, in spite of major recent progress in this context. The photon lifetime is only $0.6 \mathrm{ps}$ for $Q=1000$ and $\lambda=1 \mu \mathrm{m}$. Microspheres constitute a much different class of 3D cavities; large diameter $(\sim 100 \mu \mathrm{m})$ microspheres exhibit $Q$ 's as large as $10^{9}$, at the expense of a much larger effective volume $\left(V \sim 1000(\lambda / n)^{3}\right)$.

To conclude, much different approaches can be used to build high- $Q$, low- $V$ $3 \mathrm{D}$ dielectric microcavities. The variety in their design and in their optical properties contributes to the richness of this research field since different cavities are better suited for different applications. For instance, low-loss microspheres can be used to build extremely low-threshold lasers $[6,31]$, whereas low- $V$ semiconductor microcavities have a strong potential for the demonstration of SE rate enhancement, nearly-single mode SE coupling, or vacuum Rabi flopping, as discussed in the next sections, provided a convenient solid-state emitter (such as a single QD at low temperature) is implemented.

\section{Strong-coupling on single QDs?}

Owing to the richness of the CQED developments on atoms in the strong coupling regime [1], and to the potential application of entangled cavity/emitter 
state as $Q$-bits for quantum computing [32], it is quite interesting to discuss first whether this regime can be achieved for single QDs in semiconductor cavities.

Ideally, this regime occurs when a single localized emitter, initially in its excited state, is resonantly coupled to a single empty mode of a lossless microcavity. SE becomes a reversible process, since an emitted photon is reabsorbed before it can leave the cavity. The system experiences a Rabi oscillation at the angular frequency $\Omega$, given by

$$
\hbar \Omega=\left|d \cdot \varepsilon\left(r_{\mathrm{e}}\right)\right|
$$

where $r_{\mathrm{e}}$ is the emitter location, $\varepsilon$ - the field for one photon in the cavity mode, and $\boldsymbol{d}$ - the electric dipole of the emitter. Eigenmodes of the coupled cavity-emitter system are then two entangled states, whose energies are separated by $2 \hbar \Omega$ (so-called vacuum Rabi splitting (VRS)).

In practical cases, this regime is observed only if all dephasing mechanisms are slower than the Rabi oscillation. This is in particular the case for photon escape (cavity relaxation) or for an eventual radiative coupling of the emitter to additional leaky cavity modes (emitter relaxation). It is clear that in the solid-state other dephasing mechanisms can be dominant for the emitter. A simple criterion for the observation of the VRS in presence of decoherence is

$$
2 \hbar \Omega>\Delta E_{\mathrm{em}}+\Delta E_{\mathrm{cav}}
$$

where the $\Delta E$ 's design the emitter and cavity linewidths.

We compare therefore in Fig. 3 the VRS to the mode and QD emission linewidths; we assume that the QD is located at an antinode of the mode*. Thanks

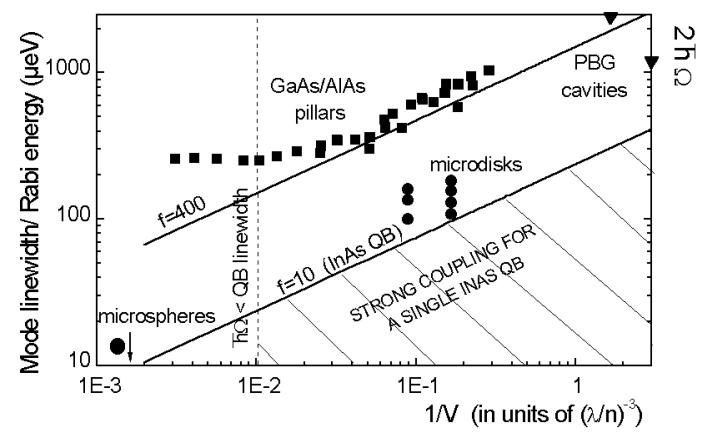

Fig. 3. Mode linewidths reported for state-of-the-art 3D micropillars [7], microdisks [8], microspheres [6], and photonic bandgap microcavities [29] are plotted as a function of the inverse effective volume of the cavity, and compared to the VRS, calculated for a single QD at the antinode of the mode.

*The norm of $\varepsilon$ at the mode antinode is related to $V$ by $|\varepsilon|_{\max }=\sqrt{\hbar \omega / 2 V \varepsilon_{0} n^{2}}$, where $n$ is the refractive index at the emitter's location. 
to the combination of a strong dipole and of a small cavity volume, large values of VRS (a few hundred of $\mu \mathrm{eV}$ 's) are well within reach of semiconductor microcavities. In particular, the VRS is then much larger than the QD linewidth at low temperature $(<30 \mu \mathrm{eV})$. However, their cavity losses are still too strong, especially for photonic bandgap microcavities and for micropillars. By contrast, silica microspheres display a very large $Q$ (up to $10^{9}$ ), but their mode volume is so large that the estimated Rabi energy for a single QD $(2 \mu \mathrm{eV})$ is much smaller than the QD linewidth. These results illustrate how the quest for the strong-coupling regime entails a complex trade-off between $Q$ and $V$ microcavity figures of merit.

In this context, wet-etched microdisks [8] appear as the most promising system at the moment since they combine a small volume $\left(V \sim 6(\lambda / n)^{3}\right)$ and a high $Q$ $(\sim 12000)$. Recent calculations show however that optimized 2D photonic bandgap microcavities might sustain modes with $Q$ 's around 30000 , well inside the strong coupling domain in Fig. 1 [33]. We therefore think that both systems might allow us to observe the strong-regime for single InAs QDs in a not too distant future. Some attention should also be given to other QDs displaying a larger oscillator strength $(f>300)$, such as those formed by interface monolayer fluctuations in quantum wells. Values of oscillator strength as large as 300 are predicted for the localized exciton trapped on large terraces, which could in principle allow us to observe more easily the strong coupling regime in microdisks, photonic bandgap cavities and state-of-the-art micropillars [34].

\section{Spontaneous-emission enhancement and single mode emission}

As shown in the previous section, QDs in 3D cavities are in the weak coupling regime; spontaneous emission is an irreversible process. In this regime, it is still possible to tailor to a large extent the $\mathrm{SE}$ rate of a radiating dipole, as first proposed by Purcell [35], by changing the density of modes to which it is coupled. SE enhancement (the so-called "Purcell effect") has been the first CQED effect to be observed on atoms; employing QDs as "artificial atoms" has been the key for its observation in monolithic microcavities [7-11]. To understand this, let us recall that the famous formula written by Purcell

$$
F_{\mathrm{p}}=\frac{3 Q(\lambda / n)^{3}}{4 \pi^{2} V}
$$

expresses the $\mathrm{SE}$ rate of an ideal emitter into one of the discrete confined modes of a $3 \mathrm{D}$ cavity, referenced to its overall $\mathrm{SE}$ rate in free-space. In this sense, $F_{\mathrm{p}}$ is a pure cavity figure of merit, which only depends on the wavelength $\lambda$, refractive index $n$ of the cavity material, effective volume $V$, and resonance factor $Q$ of the confined mode. This ideal emitter must be localized at the antinode of the confined mode, with its electric dipole aligned with the local electric field. Furthermore, it must be quasi-monochromatic on the scale of the mode linewidth and placed spectrally on perfect resonance with the cavity mode, i.e. at the maximum of the spectral 
density of modes of the cavity. If the quasi-monochromaticity requirement is not satisfied, $1 / Q$ must be replaced in expression (1) by $1 / Q+1 / Q_{e}$, where the quality factors $Q_{\mathrm{e}}$ of the emitter is defined, as for the cavity mode, by the ratio of $\lambda$ by its linewidth $\Delta \lambda$. In other words, the Purcell effects is washed out for spectrally broad emitters, and $Q_{\mathrm{e}}$ defines, for a given emitter, the upper bound for useful cavity $Q$ 's. Thanks to their narrow emission line, InAs QDs allow us to exploit fully the potentialities of high $Q$ microcavities (up to 20000 typically).

Experimentally, the Purcell effect has been first observed using direct time-resolved measurements on collections of QDs in micropillars $(\times 5[7])$, microdisks $(\times 13[8])$ and VCSEL-like oxide-apertured microcavities $(\times 2.3[9])$. In these experiments, the apparent SE rate enhancement factor is much smaller than $F_{\mathrm{p}}$, due to the random spectral and spatial distribution of the QDs with respect to the cavity mode. A statistical averaging of the magnitude of the Purcell effect permits us to account quantitatively for this result $[7,8]$.

More recently, experiments have also been conducted on single QDs in micropillars [9] or microdisks [11]. We show here in Fig. 4, as an example, typical time-resolved microPL data obtained on a $1 \mu \mathrm{m}$ diameter micropillar containing isolated QDs. A few sharp lines appear on the microPL spectrum under weak excitation conditions; since they all exhibit a linear power dependence, they correspond to "exciton lines" of different QDs. A reference PL spectrum obtained under strong excitation is all shown. In this regime, QD confined states are filled, and each QD exhibits a broadband emission, so that the PL spectrum reflects then the modal structure of the micropillar. For such a pillar, it is possible to compare directly out-of-resonance QDs, which are only coupled to leaky pillar modes, and

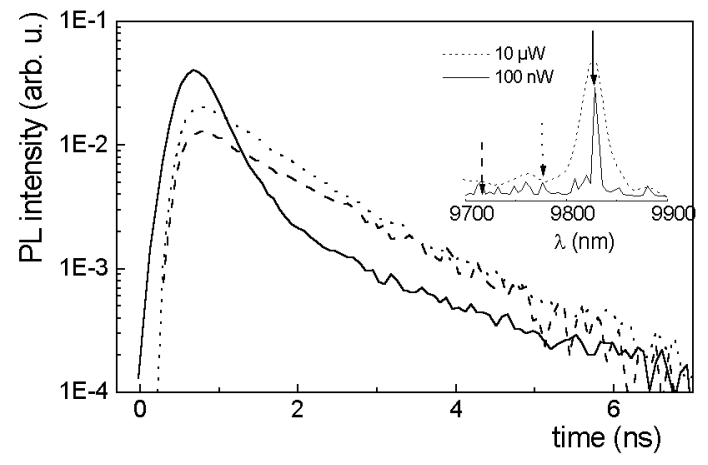

Fig. 4. Time resolved PL profiles for three isolated QDs, either placed on-resonance (solid line) or out-of-resonance (dotted and dashed lines) with the fundamental cavity mode of a $1 \mu \mathrm{m}$ diameter micropillar $\left(Q=400, F_{\mathrm{p}}=6\right)$. Inset: PL is used to probe both the distribution of isolated QDs (weak excitation) and the cavity mode (strong excitation). 
QDs in resonance, which are also resonantly coupled to the fundamental cavity mode. In spite of the moderate $F_{\mathrm{p}}(\sim 6)$ of the pillar under study, a three-fold selective enhancement of the SE rate is observed for the QD in resonance, whereas the lifetime remains much comparable to QDs in a bulk GaAs matrix $(\sim 1.3 \mathrm{~ns})$ for out-of-resonance QDs. We have observed even stronger effects $(\times 10)$ with micropillars with a higher Purcell factor using a different experimental approach, based on the study of the saturation of the QD emission under c.w. excitation. These results confirm that, as expected, the magnitude of the Purcell effect can be larger for a single QD than for a collection of QDs, provided the single QD is reasonably well matched spectrally and spatially to the cavity mode.

Until now, the Purcell effect has been observed only at relatively low temperatures $(T<50 \mathrm{~K})$. The significant broadening of the $\mathrm{QD}$ emission line due to the electron-phonon interaction ( 5 to $10 \mathrm{meV}$ at $300 \mathrm{~K}$ ) is a major problem in view of a potential application at room temperature, e.g. for the development of high-frequency light emitting diodes. At least in a near future, the Purcell effect will most likely be used not for changing SE dynamics, but as a way to collect efficiently the $\mathrm{SE}$ of an isolated QD in a single-photon source $[7,36]$.

Since the Purcell effect enhances selectively the QD emission into the resonant cavity mode, most photons are funneled into that specific mode. Considering for instance the QD in resonance in Fig. 4, we see that its SE rate into leaky modes (given by the $\mathrm{SE}$ rate of off-resonance QDs) is around $1 / 1.3 \mathrm{~ns}^{-1}$, whereas its total $\mathrm{SE}$ rate is $1 / 0.4 \mathrm{~ns}^{-1}$. The fraction of the $\mathrm{SE}$ which is coupled to the (twofold polarization degenerate) fundamental cavity mode of the circular micropillar is $\beta=1-(0.4 / 1.3) \sim 0.7$. For state-of-the-art micropillars $\left(F_{\mathrm{p}}>30\right), \beta$ 's larger than 0.95 are expected for ideally matched QDs $[7,10]$.

It should be noted that unfortunately, not all photons coupled into the cavity mode will be collected. High $Q$ micropillars have low intrinsic losses and are therefore very sensitive to additional extrinsic losses. Of particular concern to us is the optical scattering due to the residual roughness of the etched sidewalls. A careful cavity design should be done in order to ensure that most emitted photons contribute to the well collimated intrinsic emission beam of the pillar to warrant an efficient collection. Simple estimates show that for a state-of-the-art roughness and optimized cavity parameters, collection efficiencies of the order of $80 \%$ can be obtained [37], which is already very appealing.

Quite interestingly, collected photons are also prepared in a given quantum state, which can be very interesting in practice. In order to illustrate this point, we present in Fig. 5 polarization-resolved PL spectra obtained on isolated QDs in an elliptical micropillar. This anisotropic shape entails a strong birefringence, which lifts the fundamental mode degeneracy present in circular micropillars. Such pillars support a well-resolved fundamental doublet of modes with crossed linear polarizations [38]. Whereas QDs in bulk GaAs have a randomly oriented dipole in the $x-y$ plane, single QDs in resonance exhibit here a very strong linear polarization 


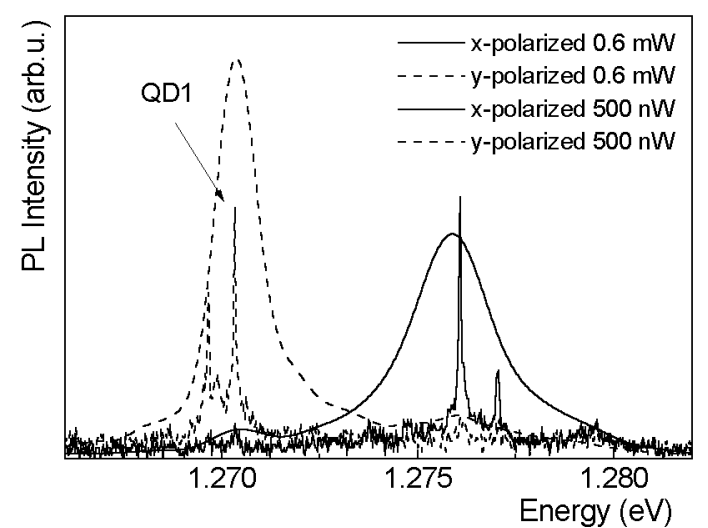

Fig. 5. Polarization-resolved microPL spectra for an elliptical micropillar $(1.5 \mu \mathrm{m} \times 0.7 \mu \mathrm{m})$ containing few QDs.

degree of their emission (up to $90 \%$ for the best QDs), imposed by the resonant cavity mode to which they are coupled.

\section{A single-mode solid-state source of single photons}

A single photon source is a device able to emit light pulses containing one and only one photon in a deterministic way. Such an ideal source, if perfect, would constitute a standard for light flux as well as for the energy. However, its most important potential applications are in the field of quantum communications and quantum information processing (quantum computing schemes based on single photons have recently been proposed [39]). In particular, certain communication schemes, which are based on the encoding of the information on single photons, can be intrinsically secure [40]. Until now however, these protocols have been validated using strongly attenuated semiconductor laser diodes $(0.1$ photon on a verage per pulse), which constitute only rough approximations to a single photon source since the photon number in a pulse follows a Poisson statistics: (1) $90 \%$ of the pulses contain no photons, which obviously limits the bit rate, (2) $1 \%$ of the pulses (i.e. $10 \%$ of the useful pulses) contain more than one photon, so that unconditional security is no more warranted [1]. The development of a true and efficient solid-state source of single photons (S4P) is thus a major challenge for quantum communications.

In order to warrant the emission of single photons, the $\mathrm{S} 4 \mathrm{P}$ should implement a single solid-state emitter. $F$-centers [41], molecules [42], and semiconductor QDs $[43,44]$ have recently demonstrated emission of photons one by one. Since the SE of an emitter of sub-wavelength size is naturally nearly omnidirectional, a microcavity should be used to collect efficiently the single photons. Unlike $F$-centers and 
molecules, whose emission lines are very broad spectrally, QDs at low temperature allow us to take benefit of the Purcell effect in microcavities. As shown in the previous section, it is possible to collect most photons, and to prepare them in a given state (the same spatial mode, the same polarization) with anisotropic micropillars, which is very interesting for an application to quantum communications. Before encoding the information on the polarization of single photons indeed, it is necessary to prepare them in a given initial state. For an unpolarized single photon source, this preparation stage would result in the loss of at least $50 \%$ of the photons.

Unlike single atoms, nothing intrinsic ensures an antibunching of the photons emitted by a single QD. It is indeed possible to store in a QD several electron-hole pairs, which can recombine and emit photons separated by arbitrarily short time delays. Some particular excitation/collection protocol must be used to ensure the preparation of single photons. A first approach relying on Coulomb blockade has been demonstrated [45]; the controlled electrical injection of a single electron and of a single hole results in the emission of a single photon. This approach seems however restricted to very low temperature $(<0.1 \mathrm{~K}$ until now $)$ and is difficult to combine with the implementation of a microcavity. It is also possible to take benefit from the strong Coulomb interaction between trapped carriers in a more simple way [36]. When several $(N)$ electron-holes pairs are injected into a QD, $N$ photons are sequentially emitted during the relaxation of the QD toward its ground state. These photons are emitted at different energies, due to the Coulomb interaction between trapped carriers. It is therefore possible to select the "last emitted" photon by using a simple spectral filtering.

The validity of this simple operation principle has been demonstrated recently by several groups, on the basis of photon correlation experiments [43, 44]. We present in Fig. 6 a correlation histogram obtained using a conventional HanburryBrown and Twiss (HBT) set-up for the $X$ emission line of a QD placed isolated in a mesa structure, for a pulsed optical pumping. The typical number of injected electron-hole pair per pulse is estimated to be $\sim 0.5$ from a comparison with the excitation power which allows us to saturate the $X$ line. The peaks in the histogram reflect the pump repetition rate at $82 \mathrm{MHz}$. For a coherent source (attenuated laser) all correlation peaks have the same area. Quite remarkably, the central correlation peak around $t=0$ is absent for the $X$ emission of the QD, which proves that a single photon at most is emitted in the $X$ line for each excitation pulse. If it was not the case indeed, some excitation pulses would lead to the emission of two photons at least and (possibly) to detection events on both detectors separated by a short delay of the order of the radiative lifetime, which would contribute to the central correlation peak.

In a complementary experiment, we have also performed cross-correlation between $X$ and $X X$ photons for a pulsed optical pumping. We place for that purpose one monochromator on each arm of the HBT set-up in order to select $X X$ 


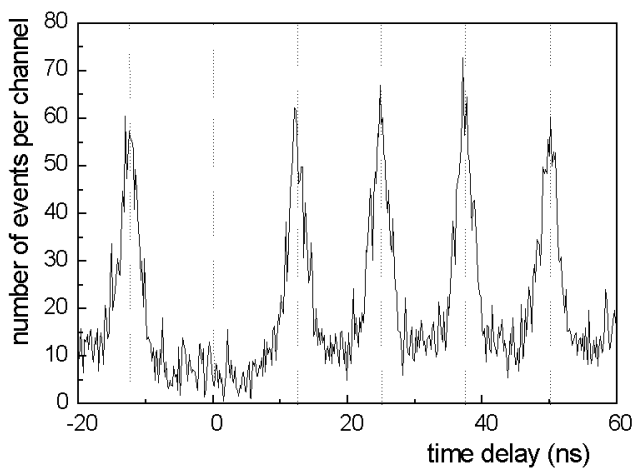

Fig. 6. Histogram of the time interval between detection events for the $X$ line of a single QD in a mesa structure under pulsed excitation $(T=8 \mathrm{~K})$. Cursors are separated by the excitation laser repetition period.

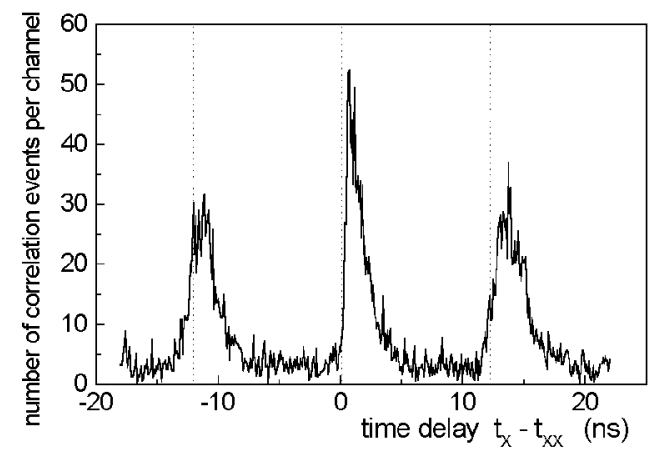

Fig. 7. Histogram of the time interval between detection events of $X$ and $X X$ photons. Cursors are separated by the excitation laser repetition period.

photons on one arm and $X$ photons on the second one. The outputs of the two detectors serve as start $(X X)$ and stop $(X)$ signals for the time-interval measurement. The central peak in the cross-correlation function shown in Fig. 7, which corresponds to the detection of a $X-X X$ photon pair generated by the same excitation pulse, displays a remarkable asymmetry. It exhibits a very sharp rise at $t=0$, followed by an exponential decay. This decay reflects the radiative recombination of the exciton, since after emission of a $X X$ photon (at $t=0$ ), the QD contains a single exciton. For negative times, the number of coincidences drops down to the floor level related to accidental coincidences due to some background emission in the PL spectrum and to the dark noise of the detectors: this shows that $X$ photons are always emitted after $X X$ photons and never before during the sequential de-excitation of the $\mathrm{QD}$. This behavior is very similar to radiative quantum cascades in atoms [46]. Other correlation peaks correspond to $X$ and $X X$ photons generated by different excitation pulses. Though they retain some of the asymmetry of the central peak, their rise is not so abrupt due to the jitter in 
the $X X$ photon detection (due to the biexciton lifetime) with respect to the laser pulses.

This set of experiments shows that QDs can be used as a triggered source of single photons as well as of photon pairs displaying strong quantum correlations. The preparation scheme works as long as the spectral separation between the QD emission lines is larger than their linewidth, in order to permit a clean spectral filtering. For InAs QDs, operation temperatures as large as $77 \mathrm{~K}$ are therefore well within reach. For II-VI QDs such as CdTe/ZnTe [12] or CdSe/ZnSe [13], the splitting between $X$ and $X X$ lines is respectively of the order of 10 and $20 \mathrm{meV}$, so that room temperature operation could most likely be achieved.

This preparation scheme is also compatible with the implementation of a microcavity to collect efficiently the single photons. We use here pillar microcavities, whose fundamental mode exhibits a collimated emission in the far field, well suited for applications. Photon correlation experiments have been performed for several QDs in resonance in a pillar microcavity such as the QD labeled "QD1" in Fig. 5. Due to the presence of some background emission due to nearby QDs, a non-zero central correlation peak is observed. Its area is however five times smaller than other peaks, which demonstrates that the probability of emitting two photons or more is five times smaller than for an attenuated laser source which would have the same photon flux. This probability should become as small as for QDs in mesas if the number of (useless) QDs in the micropillar is further reduced. Quite remarkably, the collected photon flux is about 60 times higher for the QD in the micropillar than for isolated QDs in mesas. A precise quantitative experimental estimate of the photon flux is under way; preliminary results show that, in agreement with theoretical predictions, collection efficiencies well above $50 \%$ will most likely be obtained.

\section{Conclusion}

Thanks to their very specific optical properties resulting from the strong three-dimensional confinement of trapped carriers, QDs display unique assets for the generation of quantum states of light and for solid-state CQED. Besides the Purcell effect (and related properties such as nearly single-mode emission) QDs in $3 \mathrm{D}$ semiconductor microcavities will much likely permit the observation of other CQED effects in the solid state, such as the strong coupling regime discussed in this paper, as well as non-linear effects with a few photons or thresholdless lasing. In the weak coupling regime, a single QD in resonance in an anisotropic micropillar constitutes an efficient single-mode S4P. This optoelectronic device is the first of a novel class of semiconductor devices based on a CQED effect.

\section{Acknowledgments}

This work has been partially supported by the IST-FET "S4P" project of the European commission. The authors thank V. Thierry-Mieg, L. Manin, E. Costard, 
C. Dupuis, L. Couraud for their contributions to sample growth and microcavity processing.

\section{References}

[1] For recent reviews, see Confined Electron Systems: Fundamentals and Applications, Eds. H. Benisty, J.M. Gérard, R. Houdré, J. Rarity, C. Weisbuch, Springer, Heidelberg 1999.

[2] S. Haroche, D. Kleppner, Physics Today 42, 24 (1989).

[3] C. Weisbuch, M. Nishioka, A. Ishikawa, Y. Arakawa, Phys. Rev. Lett. 69, 3314 (1992)

[4] H. De Neve, J. Blondelle, P. Van Daele, P. Demeester, R. Baets, Appl. Phys. Lett. 70, 799 (1997).

[5] D.L. Huffaker, D.G. Deppe, Appl. Phys. Lett. 71, 1449 (1997); A.E. Bond, D.P. Dapkus, J.D. O’Brien, IEEE Photonics Technol. Lett. 10, 1362 (1998).

[6] V. Sandoghdar, F. Treussart, J. Hare, V. Lefèvre-Seguin, J.-M. Raimond, S. Haroche, Phys. Rev. A 54, R1777 (1996).

[7] J.M. Gérard, B. Sermage, B. Gayral, E. Costard, V. Thierry-Mieg, Phys. Rev. Lett. 81, 1110 (1998).

[8] B. Gayral, J.M. Gérard, B. Sermage, A. Lemaítre, C. Dupuis, Appl. Phys. Lett. $78,2828(2001)$.

[9] L.A. Graham, D.L. Huffaker, D.G. Deppe, Appl. Phys. Lett. 74, 2408 (1999).

[10] G. Solomon, M. Pelton, Y. Yamamoto, Phys. Rev. Lett. 86, 3903 (2001).

[11] A. Kiraz, P. Michler, C. Becher, B. Gayral, A. Imamoglu, L. Zhang, E. Hu, Appl. Phys. Lett. 78, 3932 (2001).

[12] L. Besombes, K. Kheng, D. Martrou, Phys. Rev. Lett. 85, 425 (2000).

[13] V.D. Kulakovskii, G. Bacher, R. Weigand, T. Kümmell, A. Forchel, E. Borovitskaya, K. Leonardi, D. Hommel, Phys. Rev. Lett. 82, 1780 (1999).

[14] K. Brunner, G. Abstreiter, G. Böhm, G. Tränkle, G. Weimann, Phys. Rev. Lett. 73, 1138 (1994).

[15] M.V. Artemyev, U. Woggon, Appl. Phys. Lett. 76, 1353 (2000).

[16] X. Fan, P. Palinginis, S. Lacey, H. Wang, M.C. Lonergan, Opt. Lett. 25, 1600 (2000).

[17] L. Goldstein, F. Glas, J.Y. Marzin, M.N. Charasse, G. Le Roux, Appl. Phys. Lett. 47, 1099 (1985).

[18] J.Y. Marzin, J.M. Gérard, A. Izraël, D. Barrier, G. Bastard, Phys. Rev. Lett. 73, 716 (1994)

[19] J.L. Spithoven, J. Lorbacher, I. Manke, F. Heinrichsdorff, A. Krost, D. Bimberg, M. Dähne-Prietsch, J. Vac. Sci. Technol. B 17, 1632 (1999).

[20] L. Landin, M. Miller, M.E. Pistol, C.E. Pryor, L. Samuelson, Science 280, 262 (1998).

[21] J.M. Gérard, A. Lemaítre, B. Legrand, A. Ponchet, B. Gayral, V. Thierry-Mieg, J. Cryst. Growth 201/202, 1109 (1999). 
[22] R.J. Warburton, C.S. Dürr, K. Karrai, J.P. Kotthaus, G. Medeiros-Ribeiro, P.M. Petroff, Phys. Rev. Lett. 79, 5282 (1997).

[23] J.M. Gérard, J.B. Génin, J. Lefebvre, J.M. Moison, N. Lebouché, F. Barthe, J. Cryst. Growth 150, 351 (1995).

[24] J.M. Gérard, O. Cabrol, B. Sermage, Appl. Phys. Lett. 68, 1113 (1996).

[25] S.L. McCall, A.F.J. Levi, R.E. Slusher, H.H. Houch, N.A. Whittaker, A.C. Gossard, J.H. English, Appl. Phys. Lett. 60, 289 (1992).

[26] E. Yablonovitch, J. Opt. Soc. Am. B 10, 283 (1993).

[27] J. Jewell, A. Scherer, S.L. McCall, Y.H. Lee, S. Walker, J.P. Harbison, L.T. Florez, Electron. Lett. 25, 1123 (1989).

[28] J.M. Gérard, D. Barrier, J.Y. Marzin, R. Kuszelewicz, L. Manin, E. Costard, V. Thierry-Mieg, T. Rivera, Appl. Phys. Lett. 69, 449 (1996).

[29] O.J. Painter, A. Husain, A. Scherer, J.D. O'Brien, I. Kim, D. Dapkus, J. Lightwave Technol. 17, 2082 (1999).

[30] S. Noda, K. Tomoda, N. Yamamoto, A. Chutinan, Science 289, 604 (2000).

[31] M. Pelton, Y. Yamamoto, Phys. Rev. A 59, 2418 (1999).

[32] A. Imamoglu, D. Awschalom, G. Burkard, D.P. Di Vicenzo, D. Loss, M. Sherwin, A. Small, Phys. Rev. Lett. 83, 4204 (1999).

[33] J. Vuckovic, M. Lonkar, H. Mabuchi, A. Scherer, to appear in Phys. Rev. E (2001).

[34] L.C. Andreani, G. Panzarini, J.M. Gérard, Phys. Rev. B. 60, 13276 (1999).

[35] E.M. Purcell, Phys. Rev. 69, 681 (1946).

[36] J.M. Gérard, B. Gayral, J. Lightwave Technol. 17, 2089 (1999).

[37] W. Barnes, G. Björk, J.M. Gérard, P. Jonsson, V. Zwiller, to appear in Eur. J. Phys.

[38] B. Gayral, J.M. Gérard, Appl. Phys. Lett. 72, 1421 (1998).

[39] E. Knill, R. Laflamme, G. Millburn, Nature 409, 46 (2001).

[40] C.H. Bennett, F. Bessette, G. Brassard, L. Salvail, J. Smolin, J. Cryptology 5, 3 (1992)

[41] C. Kurtsiefer, S. Mayer, P. Zarda, H. Weinfurter, Phys. Rev. Lett. 89, 290 (2000).

[42] Th. Basché, W.E. Moerner, M. Orrit, H. Talon, Phys. Rev. Lett. 69, 1516 (1992).

[43] P. Michler, A. Kiraz, C. Becher, W. Schoenfeld, P.M. Petroff, L. Zhang, E. Hu, A. Imamoglu, Science 290, 2282 (2000).

[44] C. Santori, M. Pelton, G. Solomon, Y. Dale, Y. Yamamoto, Phys. Rev. Lett. 86, 1502 (2001).

[45] J. Kim, O. Benson, H. Kan, Y. Yamamoto, Nature 397, 500 (1999).

[46] A. Aspect, G. Roger, S. Reynaud, J. Dalibard, C. Cohen-Tannoudji, Phys. Rev. Lett. 45, 617 (1980). 